\title{
Decision-Making Models Based on Satisfaction Degree with Incomplete Hesitant Fuzzy Preference Relation
}

Jian Li

Nanning Normal University

Li-li Niu

Guangxi University

Qiongxia Chen ( $\sim$ qxchen@nnnu.edu.cn )

Nanning Normal University

Zhong-xing Wang

Guangxi University

\section{Research Article}

Keywords: Multi-criteria decision-making, incompletehesitant fuzzy preference relation, additive consistency, multiplicative consistency, satisfaction degree

Posted Date: August 2nd, 2021

DOl: https://doi.org/10.21203/rs.3.rs-765446/v1

License: (9) This work is licensed under a Creative Commons Attribution 4.0 International License. Read Full License

Version of Record: A version of this preprint was published at Soft Computing on February 24th, 2022. See the published version at https://doi.org/10.1007/s00500-021-06635-y. 


\title{
Decision-making models based on satisfaction degree with incomplete hesitant fuzzy preference relation
}

\author{
Jian $\mathrm{Li}^{1}$, Li-li Niu ${ }^{2}$, Qiongxia Chen ${ }^{1 *}$, Zhong-xing Wang ${ }^{3}$
}

1、School of Logistics Management and Engineering, Nanning Normal University, Nanning 530001, PR China

2、 Guangxi University Xingian College of Science and Liberal Arts, Nanning, 530005, PR China

3、School of Mathematics and Information Science, Guangxi University, Nanning, 530004, PR China

Correspondence should be addressed to Qiongxia Chen: qxchen@nnnu.edu.cn

\begin{abstract}
To address the situation where the incomplete hesitant fuzzy preference relation (IHFPR) is necessary, this paper develops decision-making models based on decision makers' satisfaction degree with IHFPR. First, the consistency measures from the perspectives of additive and multiplicative consistent IHFPR are defined based on the relationships between the IHPFRs and their corresponding priority weight vector, respectively. Second, two decision-making models are developed in view of the proposed additive and multiplicative consistency measures. The main characteristic of the constructed models are they taking into account the decision makers' satisfaction degree. The objective functions of the models are developed by maximizing the parameter of satisfaction degree. Third, a square programming model is developed to obtain the decision makers' weights by utilizing the optimal priority weight vectors information, the solution of the model is obtained by solving the partial derivatives of Lagrange function. Finally, a procedure for multi-criteria decision-making (MCDM) problems with IHFPRs is given, and an illustrative example in conjunction with comparative analysis is used to demonstrate the proposed models are feasible and efficiency for practical MCDM problems.
\end{abstract}

Keywords Multi-criteria decision-making, incomplete hesitant fuzzy preference relation, additive consistency, multiplicative consistency, satisfaction degree 


\section{Introduction}

Group decision making (GDM) is a specific type of decision problem where several/many decision makers cooperate with each other and choose the best solution from a set of possible alternatives (Rabiee, Aslani, \& Rezaei, 2021). In the GDM process, decision makers are invited to provide evaluation information by pairwise comparisons of alternatives, and at the end, a collective decision is reached by utilizing the predetermined criteria (Rodríguez, Labella, Dutta, \& Martínez, 2021). Due to the complexity of decision making environments, and the limitations of decision makers' knowledge, experience, and ability, decision makers are hesitant about certain evaluation values during assessments (Yazdani, Mohammed, Bai, \& Labib, 2021). To deal with this, Torra and Narukawa (2009) introduced the concept of the hesitant fuzzy set (HFS). Since that time, an increasing amount of research on the study of HFS has been published (Gong, Liu, You, \& Yin, 2021; X. Liu, Wang, Zhang, \& Garg, 2021; Mishra, Rani, Krishankumar, Ravichandran, \& Kar, 2021). Later, M. Xia and Xu (2013) found the advantages of hesitant fuzzy element (HFE) and introduced the concept of hesitant fuzzy preference relation (HFPR). Following the original work of M. Xia and Xu (2013), many multi-criteria decision-making (MCDM) approaches based on HFPR have been developed. A concise literature review of these approaches is presented as follows and summarized in Table 1.

According to number of fuzzy preference relation (FPR) was used to derive priority weight vector, all these MCDM approaches can be classified into four categories (Meng, Chen, \& Tang, 2020): (1) Only considers one FPR derived from HFPR (Song \& Li, 2019; Bin Zhu \& Xu, 2014a; B Zhu, Xu, \& Xu, 2014). This method also named optimistic consistency, that is, a reduced FPR with the highest consistency degree is derived from HFPR. The optimistic consistency method can reflect the highest consistency degree of HFPR, but it cannot reflect the hesitancy of decision makers. It leads to substantial information loss. (2) Based on ordered FPRs derived from normalized HFPR (H. Liu, Xu, \& Liao, 2016; Z. Zhang, Wang, \& Tian, 2015a, 2015b). This method also named normalized consistency. The normalized consistency requires that any two HFEs have an equal number of elements, if two HFEs have an unequal number of elements, a normalized process is needed. Therefore, the normalized consistency method may distort the original information provided by decision makers. (3) Based on all possible FPRs including in HFPR (Z. Zhang, Kou, \& Dong, 2018; Z. Zhang, Kou, Yu, \& Guo, 2018). This method also named average consistency. The method defines the concept of consistent HFPR used all possible FPRs, this seems too restricted. It is difficult for decision makers to provide such pairwise judgement in the actual decision-making process. (4) Based on the derived FPRs for each value 
in HFEs (Meng \& An, 2017; Tang, An, Meng, \& Chen, 2017). This method also named partial average consistency. The main feature of this method is that it considers all the evaluation information, and neither adds values into HFEs nor removes values from HFEs. Compared with (3), this method only used some possible FPRs including in HFPR.

Table 1 A summary of different consistent HFPRs

The category of different Main characteristic

Representative literature

consistent HFPRs

Optimistic consistency

Normalized consistency

Average consistency normalized HFPRs

Based on all possible FPRs including in HFPRs

average Based on the derived FPRs for each value in HFEs

Partial consistency
(Song \& Li, 2019; B Zhu, et al., 2014)

(H. Liu, et al., 2016; Z. Zhang, et al., 2015a)

(Z. Zhang, X. Kou, \& Q. Dong, 2018;

Z. Zhang, X. Kou, W. Yu, et al., 2018)

Due to the lack of knowledge and decision makers' limited expertise, it may be difficult for decision makers to provide complete preference relations over alternatives (J. Liu, Li, Huang, Liu, \& Liu, 2021; Wan, Yuan, \& Dong, 2021; Z. Zhang \& Chen, 2021a). At the end, lots of MCDM approaches have been developed to managing incomplete information (Dong, Liu, Chiclana, Kou, \& Herrera-Viedma, 2019; Meng \& Chen, 2021; Xie, Xu, Ren, \& Herrera-Viedma, 2020). According to their principles of derived priority weight vector, all these MCDM approaches can be classified into two categories: (1) deriving the priority weight vector based on complete FPR (Z. Zhang, 2016; Z. Zhang, et al., 2015b). This method firstly obtained the missing values based on certain rules, and then derived priority weight vector from complete FPR. However, this method only apply to the situations where each alternative is compared at least once (Ding, et al., 2020). (2) Deriving the priority weight vector based on incomplete FPR (Xu, Chen, Rodríguez, Herrera, \& Wang, 2016; Z. Zhang, X. Kou, W. Yu, et al., 2018). When implementing this method, the priority weight vector can be derived by using some programming models. It does not need to derive the missing values, and have ability to handle the case where ignored alternatives exist (Tang, Chen, \& Meng, 2019).

Since the development of HFPRs and decision makers may provide incomplete preference relations over alternatives. It is necessary to develop some approaches to managing incomplete information for HFPRs. For that, 
several MCDM approaches based on incomplete HFPR (IHFPR) have been proposed (Khalid \& Beg, 2017; Xu, et al., 2016; Z. Zhang, 2016; Z. Zhang, X. Kou, W. Yu, et al., 2018; Z. Zhang, et al., 2015b). For example, Z. Zhang, et al. (2015b) developed two methods to estimate the missing elements in an IHFPR based on the properties of additive consistent HFPR, while Z. Zhang (2016) in a similarly way to estimate the missing elements based on the properties of multiplicative consistent IHFPR. Xu, et al. (2016) developed two goal programming models to derive the priority weights from an IHFPR based on additive and multiplicative consistency, respectively. Z. Zhang, X. Kou, W. Yu, et al. (2018) proposed an approach to deriving a priority weight vector from an IHFPR using the logarithmic least squares method.

The concept of IHFPR has been introduced, and several scholars have studied some MCDM methods under incomplete hesitant evaluation environments. However, there are still some important issues need to be solved. (1) The concept of additive and multiplicative consistent IHFPR. As HFPR, additive and multiplicative consistent IHFPR develops in considering one FPR derived from IHFPR may lead to information loss (Z. Zhang, 2016), develops in considering ordered FPRs derived from normalized IHFPR may distort the preference information (Z. Zhang, 2016; Z. Zhang, et al., 2015b), and develops in considering all possible FPRs in IHFPR seems too restriction (Z. Zhang, X. Kou, W. Yu, et al., 2018). (2) Almost MCDM methods with IHFPR focus on checking and improving the consistency and consensus (Meng, et al., 2020). The priority vector follow the consistent IHFPR can obtain a reason ranking. However, for improving the consistency and consensus level may lead to destroy the original evaluation information (Peijia Ren, Xu, Wang, \& Zeng, 2021). Moreover, the disobedience and non-cooperation behaviors may be ignored in above mentions MCDM methods (H. Zhang, Palomares, Dong, \& Wang, 2018). Besides, these methods seldom consider the satisfaction degree of decision makers.

To eliminate above mention defects, the consistency measures from the perspectives of additive and multiplicative consistent IHFPR are defined based on the relationships between the IHPFRs and their corresponding weight vector, respectively. And two decision-making models are developed in view of the proposed additive and multiplicative consistency measures. The primary contributions of this study are summarized as follows.

(1) To overcome the shortcoming of additive consistent and multiplicative consistent IHFPR develops in considering a FPR derived from IHFPR, ordered FPRs derived from normalized IHFPR, and all possible FPRs including in IHFPR, a new concept of additive and multiplicative consistent IHFPR is proposed, respectively. 
(2) To consider the satisfaction degree of decision makers, two decision-making models are developed based on the proposed additive and multiplicative consistency measures. The main characteristic of the constructed models is that the objective functions of the models are obtained by maximizing the parameter of satisfaction degree.

The remainder of the paper is organized as follows. In Section 2, basic concepts and operations related to FPR, HFS and HFPR are reviewed. In Section 3, the concepts of additive and multiplicative consistent IHFPR are presented, and two decision-making models are developed in view of the proposed additive and multiplicative consistency measures. In Section 4, a square programming model is developed to obtain the decision makers' weights, and a procedure for MCDM problems with IHFPR is provided. In Section 5, the proposed method is illustrated by an example, and a comparative analysis is provided. Finally, conclusions are presented in Section 6.

\section{Preliminaries}

To carry out the following research, this part briefly reviews some basic concepts, including the concepts of FPR, HFS, and HFPR.

\subsection{FPR}

Let $X=\left\{x_{1}, x_{2}, \mathrm{~L}, x_{n}\right\}$ denotes a finite set of alternatives, where $x_{i}$ represents the $i$ th alternate. Orlovsky (1978) introduced the concept of FPR to represent fuzzy judgement value.

Definition 1 (Orlovsky, 1978). A FPR on a set of alternatives $X$ is represented by a matrix $R=\left(r_{i j}\right)_{n \times n} \subset X \times X$, where $r_{i j}$ is interpreted as the degree to which alternative $x_{i}$ is preferred to $x_{j}$. Furthermore, $r_{i j}$ should satisfy the following conditions:

$$
r_{i j}+r_{j i}=1, r_{i i}=0.5 \text { for all } i, j \in N
$$

To measure the rationality of FPR, the concepts of additive and multiplicative consistent FPR were developed.

Definition 2 (Tanino, 1984). For a FPR $R=\left(r_{i j}\right)_{n \times n}$, suppose that $W=\left(w_{1}, w_{2}, \mathrm{~L}, w_{n}\right)$ is the priority weight vector derived from $R$, where $w_{i} \in[0,1]$ and $\sum_{i=1}^{n} w_{i}=1$. Then FPR is called to be additive consistency if

$$
r_{i j}=\frac{1}{2}\left(w_{i}-w_{j}\right)+0.5 \text { for all } i, j \in N .
$$

And FPR is multiplicative consistency if 


$$
r_{i j}=\frac{w_{i}}{w_{i}+w_{j}} \text { for all } i, j \in N
$$

\subsection{HFS}

To express the hesitant preference information, Torra and Narukawa (2009) introduced the concept of HFS.

Definition 3 (Torra \& Narukawa, 2009). Let $X$ be a fixed set. Accordingly, a HFS $E$ on $X$ is defined in terms of a function $h_{E}(x)$ that when applied to $X$ returns a finite subset of $[0,1]$.

To be easily understood, M. M. Xia and Xu (2011) expressed the HFS as the following mathematical symbol:

$$
E=\left\{<x, h_{E}(x)>\mid x \in X\right\},
$$

where $h_{E}(x)$ is a set of values in $[0,1]$ representing the possible membership degrees of element $x$ in $X$ to $E$, and $h_{E}(x)$ is named hesitant fuzzy element (HFE) and denoted as $h=\left\{\gamma^{s} \mid s=1,2, \mathrm{~L}, \# h\right\}, \# h$ is the number of elements including in $h$.

The number of elements in different HFEs may be different. Any two HFEs are required to have the same length when develop the MCDM methods which the evaluation values with HFEs. To this end, a normalization process is necessary.

Definition 4 (Bin Zhu \& Xu, 2014b). Let $h=\left\{\gamma^{s} \mid s=1,2, \mathrm{~L}, \# h\right\}$ be a HFE, $\gamma^{-}$and $\gamma^{+}$denote the minimum and maximum values in $h$, respectively. Then, $(1-\xi) \gamma^{-}+\xi \gamma^{+}$is called an adding value in $h$, where $\xi$ is an optimized parameter determined by decision makers' risk preference. Especially, the decision makers are pessimistic when $\xi=0$ and decision makers are optimistic when $\xi=1$.

Evidently, different forms of normalized HFEs (NHFEs) will be derived with respect to decision makers with different risk preferences. That is, the normalization process is influenced by the subjectivity of the decision makers, and different ranking values will be derived with respect to MCDM methods with different forms of NHFEs. These shortcomings have been studied by several scholars. For more detail, the readers turn to (Li, Wang, \& Hu, 2019; Meng \& An, 2017; Z. Zhang, X. Kou, W. Yu, et al., 2018). Meanwhile, an issue on obtaining ranking values that are not relied on NHFEs arises. It will be discussed in Section 3.

\subsection{HFPR}


M. Xia and Xu (2013) first proposed the concept of HFPR. However, the sequence relationships of the elements including in HFPR are needed, this leads to some complexity in actual application. To overcome this weaknesses, Xu, Cabrerizo, and Herrera-Viedma (2017) developed a new definition of HFPR that does not need to arrange the elements in descending or ascending sequence.

Definition 5 (Xu, et al., 2017). Let $X=\left\{x_{1}, x_{2}, \mathrm{~L}, x_{n}\right\}$ be a fixed set, HFPRs on $X$ is represented by a matrix $R=\left(h_{i j}\right)_{n \times n} \subset X \times X$, where $h_{i j}=\left\{\gamma_{i j}^{s} \mid s=1,2, \mathrm{~L}, \# h_{i j}\right\}$ is a HFE indicating the possible values of the preference degrees to which alternative $x_{i}$ is preferred to alternative $x_{j}$. For all $i, j \in N, h_{i j}$ should satisfy:

$$
\gamma_{i j}^{s}+\gamma_{j i}^{\# h_{i j}-s+1}=1, \gamma_{i i}=0.5, \# h_{i j}=\# h_{j i},
$$

where $\gamma_{i j}^{s}$ refers to the $s$ th element in $h_{i j}$.

Incomplete evaluations sometimes occur for many reasons, including time pressure or lack of decision maker background knowledge. Xu, et al. (2016) developed the concept of IHFPR as follows.

Definition $6\left(\mathrm{Xu}\right.$, et al., 2016). Let $X=\left\{x_{1}, x_{2}, \mathrm{~L}, x_{n}\right\}$ be a fixed set, then an IHFPR on $X$ is represented by a matrix $R=\left(h_{i j}\right)_{n \times n} \subset X \times X$, where all known HFEs $h_{i j}=\left\{\gamma_{i j}^{s} \mid s=1,2, \mathrm{~L}, \# h_{i j}\right\}$ indicating the possible values of the preference degrees to which alternative $x_{i}$ is preferred to $x_{j}$. For all $i, j \in N, h_{i j}$ should satisfy the following conditions:

$$
\gamma_{i j}^{s}+\gamma_{j i}^{\# h_{i j}-s+1}=1, \gamma_{i i}=0.5, \# h_{i j}=\# h_{j i},
$$

where $\gamma_{i j}^{s}$ refers to the $s$ th element in $h_{i j}$.

Integrating the concepts of HFPR with additive and multiplicative consistency into IHFPR, Xu, et al. (2016) developed the concepts of additive and multiplicative consistent IHFPR.

Definition 7 (Xu, et al., 2016). Let $R=\left(h_{i j}\right)_{n \times n} \subset X \times X$ be an IHFPR, where $h_{i j}=\left\{\gamma_{i j}^{s} \mid s=1,2, \mathrm{~L}\right.$, \# $\left.h_{i j}\right\}$. If $R$ satisfies the following condition:

$$
\frac{1}{2}\left(w_{i}-w_{j}\right)+0.5=\gamma_{i j}^{1} \text { or } \gamma_{i j}^{2} \text { or } \mathrm{L} \text { or } \gamma_{i j}^{\# h_{i j}},
$$

then $R$ is called additive consistent IHFPR. Where $W=\left(w_{1}, w_{2}, \mathrm{~L}, w_{n}\right)$ is the priority weight vector derived from 
$R$.

And IHFPR is multiplicative consistency if

$$
\frac{w_{i}}{w_{i}+w_{j}}=\gamma_{i j}^{1} \text { or } \gamma_{i j}^{2} \text { or } \mathrm{L} \text { or } \gamma_{i j}^{\# h_{i j}}
$$

\section{Deriving priority weight vectors from IHFPRs}

In this section, we first introduce the concepts of additive and multiplicative consistent IHFPR, and then introduce several programming models for deriving priority weight vectors from IHFPRs, which considers the satisfaction degrees of decision makers.

\subsection{Additive and multiplicative consistent IHFPR}

To further consider Definition 7, the concepts of additive and multiplicative consistent IHFPR are respectively defined on the basis of the relationships between the formula consisting of priority weights and the values including in HFEs. However, the relationships present in Eqs. (7) and (8) only consider the relationships between one priority weight formula and all the values including in IHFPR but cannot reflect the hesitancy of decision makers. It is reasonable that for every value including in IHFPR has a relationship to one priority weight formula. That is to say, the additive and multiplicative consistent IHFPR are in accordance with the derived FPRs with respect to each fixed values. On the basis of this consideration, new concepts for additive and multiplicative consistent IHFPR are defined as follows.

Definition 8. Let $R=\left(h_{i j}\right)_{n \times n} \subset X \times X$ be an IHFPR, where $h_{i j}=\left\{\gamma_{i j}^{s} \mid s=1,2, \mathrm{~L}, \# h_{i j}\right\}$. Then, $R$ is called additive consistent IHFPR if all known elements including in $R$ satisfying the following condition:

$$
\delta_{i j}\left(\frac{1}{2}\left(w_{i}^{k}-w_{j}^{k}\right)+0.5\right)=\delta_{i j}\left(\sum_{s=1}^{\# h_{i j}} \alpha_{i j}^{s} \gamma_{i j}^{s}\right),
$$

for all $i, j=1,2, \mathrm{~L}, n$, with $i<j$, where $w_{i}^{k}, k=1,2, \mathrm{~L}, \prod_{i=1}^{n-1} \prod_{j=i+1}^{n}\left(\# h_{i j}\right)^{\delta_{i j}}$ and $i=1,2, \mathrm{~L}, n$ are the priority weights such that $w_{i}^{k} \geq 0$ and $\sum_{i=1}^{n} w_{i}^{k}=1, i=1,2, \mathrm{~L}, n$ for all $k=1,2, \mathrm{~L}, \prod_{i=1}^{n-1} \prod_{j=i+1}^{n}\left(\# h_{i j}\right)^{\delta_{i j}}$. In addition, $\alpha_{i j}^{s}, s=1,2, \mathrm{~L}, \# h_{i j}$ are $\# h_{i j}$ list of $0-1$ indicator variables, which satisfy $\sum_{s=1}^{\# h_{i j}} \alpha_{i j}^{s}=1$. To make sure for each possible value $\gamma_{i_{o} j_{o}}^{s} \in h_{i j}, i_{0}<j_{0}$ and $s=1,2, \mathrm{~L}, \# h_{i j}$ has a relationship to one priority weight formula, we 
set $\alpha_{i_{o} j_{o}}^{s}=1$ for each possible value. And $\delta_{i j}$ is an indicator variable, where $\delta_{i j}=\left\{\begin{array}{l}1, \gamma_{i j} \text { is not a missing HFE } \\ 0, \gamma_{i j} \text { is a missing HFE }\end{array}\right.$.

Obviously, there are $\prod_{i=1}^{n-1} \prod_{j=i+1}^{n}\left(\# h_{i j}\right)^{\delta_{i j}}$ FPRs corresponding to $R$. For convenience in following discussion, let $l=\prod_{i=1}^{n-1} \prod_{j=i+1}^{n}\left(\# h_{i j}\right)^{\delta_{i j}}$.

In a similarly way, the concept of multiplicative consistent IHFPR is developed as follows.

Definition 9. Let $R=\left(h_{i j}\right)_{n \times n} \subset X \times X$ be an IHFPR, where $h_{i j}=\left\{\gamma_{i j}^{s} \mid s=1,2, \mathrm{~L}, \# h_{i j}\right\}$. Then, $R$ is called multiplicative consistent IHFPR if all known elements including in $R$ satisfying the following condition:

$$
\delta_{i j}\left(\frac{w_{i}^{k}}{w_{i}^{k}+w_{j}^{k}}\right)=\delta_{i j}\left(\prod_{s=1}^{\# h_{i j}}\left(\gamma_{i j}^{s}\right)^{\alpha_{i j}^{s}}\right)
$$

for all $i, j=1,2, \mathrm{~L}, n$, with $i<j$. The meanings of symbols $w_{i}^{k}, \delta_{i j}$ and $\alpha_{i j}^{s}$ are the same as those shown in Eq. (9).

Remark 1. From Definition 8 and Definition 9 can be easily found that, there are $\# h_{i j}$ equations including in Eq. (9) or Eq. (10), whereas there is only one equation including in Eq. (7) or Eq. (8). This is the difference between Xu, et al. (2016)'s concept and proposed definitions from the view of mathematical symbol. In addition, Xu, et al. (2016)'s concept is also named optimistic consistency, a reduced FPR with the highest consistency degree is obtained, whereas the proposed definitions derived $\# h_{i j}$ possible FPRs including in HFPR.

\subsection{Deriving priority weight vectors from IHFPRs}

Consistency of preference relations is related to rationality. By comparison, inconsistent preference relations often lead to misleading solutions. Therefore, developing some approaches to obtain the expected consistency level is necessary. However, only few scholars focus on optimization-based method to obtain the expected consistent IHFPR at present. Therefore, in this section, several mathematical programming models are proposed to obtain acceptable consistent IHFPR which considering the satisfaction degrees of decision makers. There are two cases including, namely deriving priority weight vectors from IHFPRs based on the additive consistency and multiplicative consistency, respectively.

\section{Case 1. Deriving priority weight vectors from IHFPRs with additive consistency}


According to the definition of additive consistent IHFPR, we obtain $\delta_{i j}\left(\frac{1}{2}\left(w_{i}^{k}-w_{j}^{k}\right)+0.5\right)=\delta_{i j}\left(\sum_{s=1}^{\# h_{i j}} \alpha_{i j}^{s} \gamma_{i j}^{s}\right)$, where $\delta_{i j}, i, j=1,2, \mathrm{~L}, n$ indicates whether $\gamma_{i j}$ is a missing value or not. The priority weights of complete additive consistent IHFPR can be derived by solving a list of equations $\delta_{i j}\left(\frac{1}{2}\left(w_{i}^{k}-w_{j}^{k}\right)+0.5\right)=\delta_{i j}\left(\sum_{s=1}^{\# h_{i j}} \alpha_{i j}^{s} \gamma_{i j}^{s}\right)$, $i, j=1,2, \mathrm{~L}, n, i<j, k=1,2, \mathrm{~L}, l$. However, the above mentioned equations do not constantly hold in general given a deviation between $\delta_{i j}\left(\frac{1}{2}\left(w_{i}^{k}-w_{j}^{k}\right)+0.5\right)$ and $\delta_{i j}\left(\sum_{s=1}^{\# h_{i j}} \alpha_{i j}^{s} \gamma_{i j}^{s}\right)$ for each possible value $\gamma_{i_{o} j_{o}}^{s} \in h_{i j}$, $i_{0}<j_{0}$ and $s=1,2, \mathrm{~L}, \# h_{i j}$. Moreover, the more $\delta_{i j}\left(\frac{1}{2}\left(w_{i}^{k}-w_{j}^{k}\right)+0.5-\sum_{s=1}^{\# h_{i j}} \alpha_{i j}^{s} \gamma_{i j}^{s}\right)$ approaches to 0 , the more valid and reasonable the priority weights are.

In this regard, we try to obtain the priority weights of IHFPR by constructing a satisfaction degree function for the decision makers with respect to the priority weights. If the IHFPR is complete consistency then the decision maker completely satisfies the priority weights $w_{i}^{k}$ and $w_{j}^{k}$, and the satisfaction degree of the decision maker is 1 ; otherwise, the satisfaction degree of the decision maker reduces. Motivated by these studies (P. Ren, Hao, Wang, Zeng, \& Xu, 2020; P. Ren, Zhu, \& Xu, 2018; Bin Zhu \& Xu, 2014a), a membership function for the satisfaction degree in incomplete hesitant fuzzy environment can be constructed as:

$$
M_{i j}\left(w^{k}\right)= \begin{cases}1+\frac{\delta_{i j}\left(\frac{1}{2}\left(w_{i}^{k}-w_{j}^{k}\right)+0.5-\sum_{s=1}^{\# h_{i j}} \alpha_{i j}^{s} \gamma_{i j}^{s}\right)}{\varsigma_{i j}}, & \text { if } \frac{1}{2}\left(w_{i}^{k}-w_{j}^{k}\right)+0.5 \leq \sum_{s=1}^{\# h_{i j}} \alpha_{i j}^{s} \gamma_{i j}^{s} \\ 1-\frac{\delta_{i j}\left(\frac{1}{2}\left(w_{i}^{k}-w_{j}^{k}\right)+0.5-\sum_{s=1}^{\# h_{i j}} \alpha_{i j}^{s} \gamma_{i j}^{s}\right)}{S_{i j}}, & \text { if } \frac{1}{2}\left(w_{i}^{k}-w_{j}^{k}\right)+0.5>\sum_{s=1}^{\# h_{i j}} \alpha_{i j}^{s} \gamma_{i j}^{s}\end{cases}
$$

where $k=1,2, \mathrm{~L}, l, \varsigma_{i j}, \varsigma_{i j}>0$ is the parameter to present the decision makers' acceptable deviation between judgement values $\delta_{i j}\left(\sum_{s=1}^{\# h_{i j}} \alpha_{i j}^{s} \gamma_{i j}^{s}\right)$ and priority weights $\delta_{i j}\left(\frac{1}{2}\left(w_{i}^{k}-w_{j}^{k}\right)+0.5\right)$ for alternative $x_{i}$ and alternative $x_{j}$. The satisfaction degree value of $M_{i j}\left(w^{k}\right)$ varies within $(-\infty, 1]$. If $M_{i j}\left(w^{k}\right)=1$, means the equation $\delta_{i j}\left(\frac{1}{2}\left(w_{i}^{k}-w_{j}^{k}\right)+0.5-\sum_{s=1}^{\# h_{i j}} \alpha_{i j}^{s} \gamma_{i j}^{s}\right)=0$ hold, indicates that the decision makers completely satisfy the priority weights; If $0<M_{i j}\left(w^{k}\right)<1$, means the absolute value inequality $\delta_{i j}\left|\frac{1}{2}\left(w_{i}^{k}-w_{j}^{k}\right)+0.5-\sum_{s=1}^{\# h_{i j}} \alpha_{i j}^{s} \gamma_{i j}^{s}\right|<\varsigma_{i j}$ hold, 
indicates that the decision makers partly satisfy the priority weights; If $M_{i j}\left(w^{k}\right) \leq 0$, means the absolute value inequality $\delta_{i j}\left|\frac{1}{2}\left(w_{i}^{k}-w_{j}^{k}\right)+0.5-\sum_{s=1}^{\# h_{i j}} \alpha_{i j}^{s} \gamma_{i j}^{s}\right| \geq \varsigma_{i j}$ hold, indicates that the decision makers does not satisfy the priority weights.

We can utilize a regular $n-1$-simplex to present the membership functions of $M_{i j}\left(w^{k}\right), i, j=1,2, \mathrm{~L}, n$, which can be denoted as follows:

$$
S X^{n-1}=\left\{w_{1}^{k}, w_{2}^{k}, \mathrm{~L}, w_{n}^{k} \mid \sum_{i=1}^{n} w_{i}^{k}=1 \text { and } w_{i}^{k} \geq 0 \text { for all } i\right\}, k=1,2, \mathrm{~L}, l .
$$

A function can be provided to synthesize all satisfaction degrees of the priority weights $w_{i}^{k}$ and $w_{j}^{k}$, $i, j=1,2, \mathrm{~L}, n$ in $S X^{n-1}$ simplex

$$
M=\min _{w^{k} \in S X^{n-1}}\left\{M_{i j}\left(w^{k}\right) \mid i, j=1,2, \mathrm{~L}, n\right\}, k=1,2, \mathrm{~L}, l .
$$

The theory of maximum minimization is utilized to guarantee the minimum satisfaction degree be not too low. Therefore, the objective function is denoted as: $\max _{i, j=1,2, \mathrm{~L}, n} \min _{w^{k} \in S X^{n-1}}\left\{M_{i j}\left(w^{k}\right) \mid i, j=1,2, \mathrm{~L}, n\right\}$, for all $k=1,2, \mathrm{~L}, l$, which can be represented by the following programming model:

$$
\begin{array}{ll}
\max & z^{k}=\varphi \\
\text { s.t. } & \left\{\begin{array}{l}
M_{i j}\left(w^{k}\right) \geq \varphi \\
w^{k} \in S X^{n-1} \\
i, j=1,2, \mathrm{~L}, n, i \neq j
\end{array} .\right.
\end{array}
$$

Substituting equation (11) into equation (14), for each possible value $\gamma_{i_{o} j_{o}}^{s} \in h_{i j}$, with $i_{0}<j_{0}$ for each $s=1,2, \mathrm{~L}, \# h_{i j}$, the mathematical programming model can be expressed in detail as: 


$$
\begin{array}{ll}
\max & z^{k}=\varphi \\
\text { s.t. } & \left\{\begin{array}{l}
\varsigma_{i j}+\delta_{i j}\left(\frac{1}{2}\left(w_{i}^{k}-w_{j}^{k}\right)+0.5-\sum_{s=1}^{\# h_{i j}} \alpha_{i j}^{s} \gamma_{i j}^{s}\right) \geq \varphi \varsigma_{i j} \\
\varsigma_{i j}-\delta_{i j}\left(\frac{1}{2}\left(w_{i}^{k}-w_{j}^{k}\right)+0.5-\sum_{s=1}^{\# h_{i j}} \alpha_{i j}^{s} \gamma_{i j}^{s}\right) \geq \varphi \varsigma_{i j} \\
\sum_{i=1}^{n} w_{i}^{k}=1, \quad k=1,2, \mathrm{~L}, l \\
\sum_{s=1}^{\# h_{i j}} \alpha_{i j}^{s}=1 \\
\alpha_{i j}^{s}=0 \vee 1, \quad s=1,2, \mathrm{~L}, \# h_{i j} \\
\delta_{i j}=0 \vee 1 \\
\alpha_{i_{0} j_{0}}^{s}=1, \quad i_{0}<j_{0} \\
i, j=1,2, \mathrm{~L}, n, i \neq j
\end{array}\right.
\end{array}
$$

Suppose the decision makers provide the preference degree $\varsigma_{i j}$ with the equal significance, that is, $\varsigma_{i j}=\varsigma$, for all $i, j=1,2, \mathrm{~L}, n$, in this case, the mathematical programming model can be further simplified to:

$$
\begin{array}{ll}
\max \quad & z^{k}=\varphi \\
\text { s.t. } & \left\{\begin{array}{l}
\varsigma+\delta_{i j}\left(\frac{1}{2}\left(w_{i}^{k}-w_{j}^{k}\right)+0.5-\sum_{s=1}^{\# h_{i j}} \alpha_{i j}^{s} \gamma_{i j}^{s}\right) \geq \varphi \varsigma \\
\varsigma-\delta_{i j}\left(\frac{1}{2}\left(w_{i}^{k}-w_{j}^{k}\right)+0.5-\sum_{s=1}^{\# h_{i j}} \alpha_{i j}^{s} \gamma_{i j}^{s}\right) \geq \varphi \varsigma \\
\sum_{i=1}^{n} w_{i}^{k}=1, \quad k=1,2, \mathrm{~L}, l \\
\sum_{s=1}^{\# h_{i j}} \alpha_{i j}^{s}=1 \\
\alpha_{i j}^{s}=0 \vee 1, \quad s=1,2, \mathrm{~L}, \# h_{i j} \\
\delta_{i j}=0 \vee 1 \\
\alpha_{i_{0} j_{0}}^{s}=1, \quad i_{0}<j_{0} \\
i, j=1,2, \mathrm{~L}, n, i \neq j
\end{array}\right.
\end{array}
$$

Substituting deviation variable value $\varsigma$ into the above model, it can be easily proved that Eq. (16) is a $0-1$ programming model. Therefore, at least one priority weight vector can be derived with the maximum satisfaction degree $\varphi$.

Solving Eq. (16), a list of priority weight vectors $w^{k}, k=1,2, \mathrm{~L}, l$ can be derived. Since $w^{k}$ can be viewed as the possible priority weight vector of $R$. And based on the ideas of Z. Zhang, X. Kou, W. Yu, et al. (2018) and Wu, Zhu, Zhou, and Chen (2019). The distance between $w^{k}$ and $R$ is developed to select the best priority weight vector of $R$. 
Definition 10. Let $R=\left(h_{i j}\right)_{n \times n} \subset X \times X$ be an IHFPR, and $w^{k}=\left(w_{1}^{k}, w_{2}^{k}, \mathrm{~L}, w_{n}^{k}\right), k=1,2, \mathrm{~L}, l$ be a list of priority weight vectors derived from Eq. (16). Then, the distance between $w^{k}$ and $R$ is developed as follows:

$$
d_{1}\left(w^{k}, R\right)=\frac{1}{l} \sum_{i=1}^{n-1} \sum_{j=i+1}^{n} \delta_{i j}\left|\frac{1}{2}\left(w_{i}^{k}-w_{j}^{k}\right)+0.5-\sum_{s=1}^{\# h_{i j}} \alpha_{i j}^{s} \gamma_{i j}^{s}\right|
$$

where $l=\prod_{i=1}^{n-1} \prod_{j=i+1}^{n}\left(\# h_{i j}\right)^{\delta_{i j}}$ is the number of known value in upper triangle of IHFPR. It can be easily found that the distance $d_{1}\left(w^{k}, R\right)$ reflects the average of total the square deviation between $\frac{1}{2}\left(w_{i}^{k}-w_{j}^{k}\right)+0.5$ and $\sum_{s=1}^{\# h_{i j}} \alpha_{i j}^{s} \gamma_{i j}^{s}$ for all known elements including in $R$. It is natural that the optimal priority weight vector is the one that minimizes the deviation $d_{1}\left(w^{k}, R\right)$.

For the distance measure presents in Eq. (17), it can be proven that it satisfy the axiom of the distance measure.

Property 1. For the distance measure presents in Eq. (17), we have:

(1) non-negativity: $0 \leq d_{1}\left(w^{k}, R\right) \leq 1$;

(2) reflexivity: $d_{1}\left(w^{k}, R\right)=0 \Leftrightarrow \frac{1}{2}\left(w_{i}^{k}-w_{j}^{k}\right)+0.5=\sum_{s=1}^{\# h_{i j}} \alpha_{i j}^{s} \gamma_{i j}^{s}$;

(3) commutativity: $d_{1}\left(w^{k}, R\right)=d_{1}\left(R, w^{k}\right)$ and

(4) triangle inequality:

if $d_{1}^{1,2}\left(w^{k}, R\right)=\frac{1}{l} \sum_{i=1}^{n-1} \sum_{j=i+1}^{n} \delta_{i j}\left|\frac{1}{2}\left(w_{i}^{k, 1}-w_{j}^{k, 1}\right)+0.5-\sum_{s=1}^{\# h_{i j}} \alpha_{i j}^{s, 1} \gamma_{i j}^{s, 1}-\frac{1}{2}\left(w_{i}^{k, 2}-w_{j}^{k, 2}\right)-0.5+\sum_{s=1}^{\# h_{i j}} \alpha_{i j}^{s, 2} \gamma_{i j}^{s, 2}\right| \quad$ and $d_{1}^{2,3}\left(w^{k}, R\right)=\frac{1}{l} \sum_{i=1}^{n-1} \sum_{j=i+1}^{n} \delta_{i j}\left|\frac{1}{2}\left(w_{i}^{k, 2}-w_{j}^{k, 2}\right)-0.5+\sum_{s=1}^{\# h_{i j}} \alpha_{i j}^{s, 2} \gamma_{i j}^{s, 2}-\frac{1}{2}\left(w_{i}^{k, 3}-w_{j}^{k, 3}\right)-0.5+\sum_{s=1}^{\# h_{i j}} \alpha_{i j}^{s, 3} \gamma_{i j}^{s, 3}\right| \quad . \quad$ Then $d_{1}^{1,3}\left(w^{k}, R\right) \leq d_{1}^{1,2}\left(w^{k}, R\right)+d_{1}^{2,3}\left(w^{k}, R\right)$.

Proof: The proof of (2) and (3) is obvious, they do not appear in this study. In the following section, we only provide the proof of (1) and (4).

(1) non-negativity: for each known elements including in $R$, we have $0 \leq\left|\frac{1}{2}\left(w_{i}^{k}-w_{j}^{k}\right)+0.5-\alpha_{i j}^{s} \gamma_{i j}^{s}\right| \leq 1$, and 
$0 \leq\left|\frac{1}{2}\left(w_{i}^{k}-w_{j}^{k}\right)+0.5-\sum_{s=1}^{\# h_{i j}} \alpha_{i j}^{s} \gamma_{i j}^{s}\right| \leq \# h_{i j}$. Moreover, $0 \leq \sum_{i=1}^{n-1} \sum_{j=i+1}^{n} \delta_{i j}\left|\frac{1}{2}\left(w_{i}^{k}-w_{j}^{k}\right)+0.5-\sum_{s=1}^{\# h_{i j}} \alpha_{i j}^{s} \gamma_{i j}^{s}\right| \leq l$, then $0 \leq \frac{1}{l} \sum_{i=1}^{n-1} \sum_{j=i+1}^{n} \delta_{i j}\left|\frac{1}{2}\left(w_{i}^{k}-w_{j}^{k}\right)+0.5-\sum_{s=1}^{\# h_{i j}} \alpha_{i j}^{s} \gamma_{i j}^{s}\right| \leq 1$, that is $0 \leq d_{1}\left(w^{k}, R\right) \leq 1$.

(4) triangle inequality:

$$
\begin{aligned}
& d_{1}^{1,3}\left(w^{k}, R\right)=\frac{1}{l} \sum_{i=1}^{n-1} \sum_{j=i+1}^{n} \delta_{i j}\left|\frac{1}{2}\left(w_{i}^{k, 1}-w_{j}^{k, 1}\right)+0.5-\sum_{s=1}^{\# h_{i j}} \alpha_{i j}^{s, 1} \gamma_{i j}^{s, 1}-\frac{1}{2}\left(w_{i}^{k, 3}-w_{j}^{k, 3}\right)-0.5+\sum_{s=1}^{\# h_{i j}} \alpha_{i j}^{s, 3} \gamma_{i j}^{s, 3}\right| \\
& =\frac{1}{l} \sum_{i=1}^{n-1} \sum_{j=i+1}^{n} \delta_{i j} \mid \begin{array}{l}
\frac{1}{2}\left(w_{i}^{k, 1}-w_{j}^{k, 1}\right)+0.5-\sum_{s=1}^{\# h_{i j}} \alpha_{i j}^{s, 1} \gamma_{i j}^{s, 1}-\frac{1}{2}\left(w_{i}^{k, 2}-w_{j}^{k, 2}\right)-0.5+\sum_{s=1}^{\# h_{h j}} \alpha_{i j}^{s, 2} \gamma_{i j}^{s, 2} \\
+\frac{1}{2}\left(w_{i}^{k, 2}-w_{j}^{k, 2}\right)+0.5-\sum_{s=1}^{\# h_{i j}} \alpha_{i j}^{s, 2} \gamma_{i j}^{s, 2}-\frac{1}{2}\left(w_{i}^{k, 3}-w_{j}^{k, 3}\right)-0.5+\sum_{s=1}^{\# h_{i j}} \alpha_{i j}^{s, 3} \gamma_{i j}^{s, 3} \mid
\end{array} \\
& \quad \leq \frac{1}{l} \sum_{i=1}^{n-1} \sum_{j=i+1}^{n} \delta_{i j}\left|\frac{1}{2}\left(w_{i}^{k, 1}-w_{j}^{k, 1}\right)+0.5-\sum_{s=1}^{\# h_{i j}} \alpha_{i j}^{s, 1} \gamma_{i j}^{s, 1}-\frac{1}{2}\left(w_{i}^{k, 2}-w_{j}^{k, 2}\right)-0.5+\sum_{s=1}^{\# h_{i j}} \alpha_{i j}^{s, 2} \gamma_{i j}^{s, 2}\right| \\
& +\frac{1}{l} \sum_{i=1}^{n-1} \sum_{j=i+1}^{n} \delta_{i j}\left|\frac{1}{2}\left(w_{i}^{k, 2}-w_{j}^{k, 2}\right)+0.5-\sum_{s=1}^{\# h_{i j}} \alpha_{i j}^{s, 2} \gamma_{i j}^{s, 2}-\frac{1}{2}\left(w_{i}^{k, 3}-w_{j}^{k, 3}\right)-0.5+\sum_{s=1}^{\# h_{i j}} \alpha_{i j}^{s, 3} \gamma_{i j}^{s, 3}\right|
\end{aligned}
$$

Then

As a consequence, the priority weight vector of $R$ is developed as follows.

Definition 11. Let $R=\left(h_{i j}\right)_{n \times n} \subset X \times X$ be an IHFPR, and $w^{k}=\left(w_{1}^{k}, w_{2}^{k}, \mathrm{~L}, w_{n}^{k}\right), k=1,2, \mathrm{~L}, l$ be a list of priority weight vectors derived from Eq. (16). Then, the priority weight vector of $R$ is developed as follows:

$$
w^{k *}=\left(w_{1}^{k *}, w_{2}^{k *}, \mathrm{~L}, w_{n}^{k *}\right)=\arg \min _{w^{k}} d_{1}\left(w^{k}, R\right) .
$$

The symbol arg presents in Eq. (18) means the priority weight vector is derived from the minimum distance calculate from $d_{1}\left(w^{k}, R\right)$.

Remark 2. There are may be more than one priority weight vectors including in $\min _{w^{k}} d_{1}\left(w^{k}, R\right)$, that is to say, sometimes the solution of the Eq. (18) is not unique. In this case, the priority weight vector of $R$ is developed as average of multiple priority weight vectors:

$$
w^{k *}=\left(w_{1}^{k *}, w_{2}^{k *}, \mathrm{~L}, w_{n}^{k *}\right)=\left(\frac{1}{l_{0}} \sum_{k=1}^{l_{0}} w_{1}^{k}, \frac{1}{l_{0}} \sum_{k=1}^{l_{0}} w_{2}^{k}, \mathrm{~L}, \frac{1}{l_{0}} \sum_{k=1}^{l_{0}} w_{n}^{k}\right)
$$


where $w_{i}^{k}, k=1,2, \mathrm{~L}, n$, and it indicates that the number of priority weight vectors including in $\min _{w^{k}} d_{1}\left(w^{k}, R\right)$ is $l_{0}$.

\section{Case 2. Deriving priority weight vectors from IHFPRs with multiplicative consistency}

Similar to the idea of additive consistent IHFPR presented in case 1, the following membership function with the satisfaction degree can be constructed when we consider multiplicative consistent IHFPR.

$$
F_{i j}\left(w^{k}\right)=\left\{\begin{array}{ll}
1+\frac{\delta_{i j}\left(\frac{w_{i}^{k}}{w_{i}^{k}+w_{j}^{k}}-\prod_{s=1}^{\# h_{i j}}\left(\gamma_{i j}^{s}\right)^{\alpha_{i j}^{s}}\right)}{\varepsilon_{i j}}, & \text { if } \frac{w_{i}^{k}}{w_{i}^{k}+w_{j}^{k}} \leq \prod_{s=1}^{\# h_{i j}}\left(\gamma_{i j}^{s}\right)^{\alpha_{i j}^{s}} \\
1-\frac{\delta_{i j}\left(\frac{w_{i}^{k}}{w_{i}^{k}+w_{j}^{k}}-\prod_{s=1}^{\# h_{i j}}\left(\gamma_{i j}^{s}\right)^{\alpha_{i j}^{s}}\right)}{\varepsilon_{i j}}, & \text { if } \frac{w_{i}^{k}}{w_{i}^{k}+w_{j}^{k}}>\prod_{s=1}^{\# h_{i j}}\left(\gamma_{i j}^{s}\right)^{\alpha_{i j}^{s}}
\end{array},\right.
$$

where $k=1,2, \mathrm{~L}, l$. The meanings of symbols $w_{i}^{k}, \varepsilon_{i j}, \alpha_{i j}^{s}$ and $F_{i j}\left(w^{k}\right)$ are the same as those given in Eq. (11).

Similar to the idea of additive consistent IHFPR presented in case 1, the mathematical programming model for deriving priority weight vectors can be expressed in detail as:

$$
\begin{aligned}
& \max \quad z^{k}=\pi \\
& \text { s.t. }\left\{\begin{array}{l}
\varepsilon_{i j}+\delta_{i j}\left(\frac{w_{i}^{k}}{w_{i}^{k}+w_{j}^{k}}-\prod_{s=1}^{\# h_{i j}}\left(\gamma_{i j}^{s}\right)^{\alpha_{i j}^{s}}\right) \geq \pi \varepsilon_{i j} \\
\varepsilon_{i j}-\delta_{i j}\left(\frac{w_{i}^{k}}{w_{i}^{k}+w_{j}^{k}}-\prod_{s=1}^{\# h_{i j}}\left(\gamma_{i j}^{s}\right)^{\alpha_{i j}^{s}}\right) \geq \pi \varepsilon_{i j} \\
\sum_{i=1}^{n} w_{i}^{k}=1, \quad k=1,2, \mathrm{~L}, l \\
\sum_{s=1}^{\# h_{i j}} \alpha_{i j}^{s}=1 \\
\alpha_{i j}^{s}=0 \vee 1, \quad s=1,2, \mathrm{~L}, \# h_{i j} \\
\delta_{i j}=0 \vee 1 \\
\alpha_{i_{0}, j_{0}}^{s}=1, \quad i_{0}<j_{0} \\
i, j=1,2, \mathrm{~L}, n, i \neq j
\end{array}\right.
\end{aligned}
$$

Suppose $\varepsilon_{i j}=\varepsilon$, for all $i, j=1,2, \mathrm{~L}, n$, then, the mathematical programming model can be further simplified to: 


$$
\begin{aligned}
& \max \quad z^{k}=\pi \\
& \text { s.t. }\left\{\begin{array}{l}
\varepsilon+\delta_{i j}\left(\frac{w_{i}^{k}}{w_{i}^{k}+w_{j}^{k}}-\prod_{s=1}^{\# h_{i j}}\left(\gamma_{i j}^{s}\right)^{\alpha_{i j}^{s}}\right) \geq \pi \varepsilon \\
\varepsilon-\delta_{i j}\left(\frac{w_{i}^{k}}{w_{i}^{k}+w_{j}^{k}}-\prod_{s=1}^{\# h_{i j}}\left(\gamma_{i j}^{s}\right)^{\alpha_{i j}^{s}}\right) \geq \pi \varepsilon \\
\sum_{i=1}^{n} w_{i}^{k}=1, \quad k=1,2, \mathrm{~L}, l \\
\sum_{s=1}^{\# h_{i j}} \alpha_{i j}^{s}=1 \\
\alpha_{i j}^{s}=0 \vee 1, \quad s=1,2, \mathrm{~L}, \# h_{i j} \\
\delta_{i j}=0 \vee 1 \\
\alpha_{i_{0} j_{0}}^{s}=1, \quad i_{0}<j_{0} \\
i, j=1,2, \mathrm{~L}, n, i \neq j
\end{array} .\right.
\end{aligned}
$$

Substituting deviation variable value $\varepsilon$ into the above model, it can be easily proved that Eq. (22) is a nonlinear programming model. In this case, the optimal solution of it can be obtained by utilizing the optimization software, such as LINGO 11.0, Matlab and Mathematica.

Solving Eq. (22), a list of weight vectors $w^{k}, k=1,2, \mathrm{~L}, l$ can be derived. Since $w^{k}$ can be viewed as the possible priority weight vector of $R$. Similarly to additive consistent IHFPR, the distance between $w^{k}$ and $R$ is developed to select the best priority weight vector.

Definition 12. Let $R=\left(h_{i j}\right)_{n \times n} \subset X \times X$ be an IHFPR, and $w^{k}=\left(w_{1}^{k}, w_{2}^{k}, \mathrm{~L}, w_{n}^{k}\right), k=1,2, \mathrm{~L}, l$ be a list of priority weight vectors derived from Eq. (22). Then, the distance between $w^{k}$ and $R$ is developed as follows:

$$
d_{2}\left(w^{k}, R\right)=\frac{1}{l} \sum_{i=1}^{n-1} \sum_{j=i+1}^{n} \delta_{i j}\left|\frac{w_{i}^{k}}{w_{i}^{k}+w_{j}^{k}}-\prod_{s=1}^{\# h_{i j}}\left(\gamma_{i j}^{s}\right)^{\alpha_{i j}^{s}}\right| .
$$

The meanings of symbols $w_{i}^{k}, \delta_{i j}, \alpha_{i j}^{s}, l$ and $d_{2}\left(w^{k}, R\right)$ are the same as those given in Eq. (17).

Remark 3. Similar to the idea of Eq. (17), the proof of the axiom of the distance measure presents in Eq. (23) can be developed in a similar way.

Similarly, the priority weight vector of $R$ is developed as follows.

Definition 13. Let $R=\left(h_{i j}\right)_{n \times n} \subset X \times X$ be an IHFPR, and $w^{k}=\left(w_{1}^{k}, w_{2}^{k}, \mathrm{~L}, w_{n}^{k}\right), k=1,2, \mathrm{~L}, l$ be a list of priority weight vectors derived from Eq. (22). Then, the priority weight vector of $R$ is developed as follows: 


$$
w^{k *}=\left(w_{1}^{k *}, w_{2}^{k *}, \mathrm{~L}, w_{n}^{k *}\right)=\arg \min _{w^{k}} d_{2}\left(w^{k}, R\right) .
$$

Remark 4. Similar to the idea of additive consistent IHFPR presented in case 1, there are may be more than one priority weight vectors including in $\min _{w^{k}} d_{2}\left(w^{k}, R\right)$. In this case, the priority weight vector of $R$ is developed according to Eq. (19).

\section{Framework of MCDM procedure with IHFPRs}

In this section, the MCDM problems with IHFPRs are firstly introduced, and then an optimization model is constructed for determining the weights of decision makers. Finally, a framework of MCDM procedure with IHFPRs is introduced.

\subsection{The MCDM problems with IHFPRs}

Hesitant MCDM problems involve $m$ alternatives denoted as $A=\left\{a_{1}, a_{2}, \mathrm{~L}, a_{m}\right\}$. Each alternative is assessed based on several feature criteria. $E=\left\{e_{1}, e_{2}, \mathrm{~L}, e_{n}\right\}$ is a set of decision makers and $\lambda=\left(\lambda_{1}, \lambda_{2}, \mathrm{~L}, \lambda_{n}\right)^{T}$ is the decision makers' weight vector. We assume that the weights of decision makers are completely unknown. The evaluation of the alternative $a_{i}, i=1,2, \mathrm{~L}, m$ with respect to the feature criterion is provided by decision maker $e_{j}$, $j=1,2, \mathrm{~L}, n$, and denotes as $h=\left\{\gamma^{s} \mid s=1,2, \mathrm{~L}, \# h\right\}$, which is an IHFPR. Suppose IHFPRs provide by decision makers with additive consistency, then the priority weight vectors of each individual IHFPRs are derived according to Eq. (18) or Eq. (19); otherwise, the priority weight vectors of each individual IHFPRs are derived according to Eq. (23) or Eq. (19) if IHFPRs with multiplicative consistency.

\subsection{Calculate the weight vectors of the decision makers}

In this subsection, an optimization model is constructed to derive the weights of decision makers with complete unknown information. Considering that decision makers in the MCDM process typically construct from different knowledge backgrounds and have varied expertise in the domain area, each decision makers has different judgment values, which influences the solution differently. Therefore, each decision makers has a different importance weight when collecting the priority weight vectors. Given that the decision maker whose judgment values are far away from the collect judgment values indicates that the judgment values he/she provides are the least reliable, and the decision maker should endow a smaller weight value. By comparison, the decision maker whose judgment values are close to 
the collect judgment values indicates that the judgment values he/she provides are the most reliable, and the decision maker should endow a larger weight value. On this basis, the optimization model is constructed as follows:

$$
\begin{aligned}
& \min z=\sum_{k=1}^{n} \sum_{i=1}^{m}\left(w_{i}^{k *}-\sum_{k=1}^{n} \lambda_{k} w_{i}^{k *}\right)^{2} \\
& \text { s.t. }\left\{\begin{array}{l}
\sum_{k=1}^{n} \lambda_{k}=1 \\
0 \leq \lambda_{k} \leq 1 \\
k=1,2, \mathrm{~L}, n
\end{array}\right.
\end{aligned}
$$

As seen, Eq. (25) is a square programming model. The optimal solution of it can be obtained by utilizing the optimization software. Moreover, it can be easily found that Eq. (25) is constructed from the algorithm of least square method, according to the Lagrange multiplier method, the Lagrange function of Eq. (25) can be constructed as follows, $L(\lambda, \mu)=\sum_{k=1}^{n} \sum_{i=1}^{m}\left(w_{i}^{k *}-\sum_{k=1}^{n} \lambda_{k} w_{i}^{k *}\right)^{2}+2 \mu\left(\sum_{k=1}^{n} \lambda_{k}-1\right)$, where $\mu$ is a Lagrange multiplier. The solution of Eq.

(25) can be obtained by solving the partial derivatives of Lagrange function $L(\lambda, \mu)$, and the result is listed as follows:

$$
\lambda=\left(\lambda_{1}, \lambda_{2}, \mathrm{~L}, \lambda_{n}\right)^{T}=A^{-1}\left(B-\frac{e^{T} A^{-1} B e-e}{e^{T} A^{-1} e}\right),
$$

where $A=\left[\begin{array}{cccc}\sum_{i=1}^{m} w_{i}^{1 *} w_{i}^{1 *} & \sum_{i=1}^{m} w_{i}^{2 *} w_{i}^{1 *} & \mathrm{~L} & \sum_{i=1}^{m} w_{i}^{n^{*}} w_{i}^{1 *} \\ \sum_{i=1}^{m} w_{i}^{1 *} w_{i}^{2 *} & \sum_{i=1}^{m} w_{i}^{2 *} w_{i}^{2 *} & \mathrm{~L} & \sum_{i=1}^{m} w_{i}^{n *} w_{i}^{2 *} \\ \mathrm{M} & \mathrm{M} & \mathrm{M} & \mathrm{M} \\ \sum_{i=1}^{m} w_{i}^{1 *} w_{i}^{n *} & \sum_{i=1}^{m} w_{i}^{2 *} w_{i}^{n *} & \mathrm{~L} & \sum_{i=1}^{m} w_{i}^{n *} w_{i}^{n *}\end{array}\right], B=\left[\begin{array}{c}\sum_{k=1}^{n} \sum_{i=1}^{m} w_{i}^{k^{*}} w_{i}^{1 *} \\ \sum_{k=1}^{n} \sum_{i=1}^{m} w_{i}^{k^{*}} w_{i}^{2 *} \\ \mathrm{M} \\ \sum_{k=1}^{n} \sum_{i=1}^{m} w_{i}^{k *} w_{i}^{n *}\end{array}\right], e=(1,1, \mathrm{~L}, 1)^{T}$ and $A^{-1}$ is the inverse matrix of $A, e^{T}$ is the transpose matrix of $e$.

\subsection{Framework of MCDM procedure with IHFPRs}

The proposed decision making procedure is summarized in the following steps.

Step 1: Form individual IHFPR matrices.

According to the determine criteria and alternatives, the decision makers respectively provide their judgement matrices, and denotes as $R_{k}=\left(h_{i j, k}\right)_{m \times m} \subset X \times X, k=1,2, \mathrm{~L}, n$. 
Step 2: Derive the priority weight vectors.

Utilize Eq. (16) or Eq. (22) to obtain the priority weight vectors $w^{k}=\left(w_{1}^{k}, w_{2}^{k}, \mathrm{~L}, w_{n}^{k}\right), k=1,2, \mathrm{~L}, n$ from the individual IHFPR.

Step 3: Derive the optimal priority weight vector.

First, Utilize Eq. (17) or Eq. (23) to calculate the distance between $w^{k}$ and $R_{k}$, if there is only one priority weight vector including in Eq. (18) or Eq. (24), then the optimal priority weight vector is determined according to Eq. (18) or Eq. (24), otherwise, the optimal priority weight vector is determined according to Eq. (19).

Step 4: Determine the weights of decision makers.

The weights of decision makers are determined according to Eq. (26).

Step 5: Compute the collective optimal priority weight vector.

The collective optimal priority weight vector is determined by the following formula:

$$
\vartheta_{i}=\sum_{k=1}^{n} \lambda_{k} w_{i}^{k *}, i=1,2, \mathrm{~L}, m,
$$

where $\lambda_{k}$ is the weight of decision maker, and $w_{i}^{k *}$ is the optimal priority weight vector determines in Step 3.

Step 6: Rank the alternatives.

The ranking order of all alternatives is obtained by the value of collective optimal priority weight vector $\vartheta_{i}$, $i=1,2, \mathrm{~L}, m$.

The proposed decision making procedure is depicted in Fig. 1. 


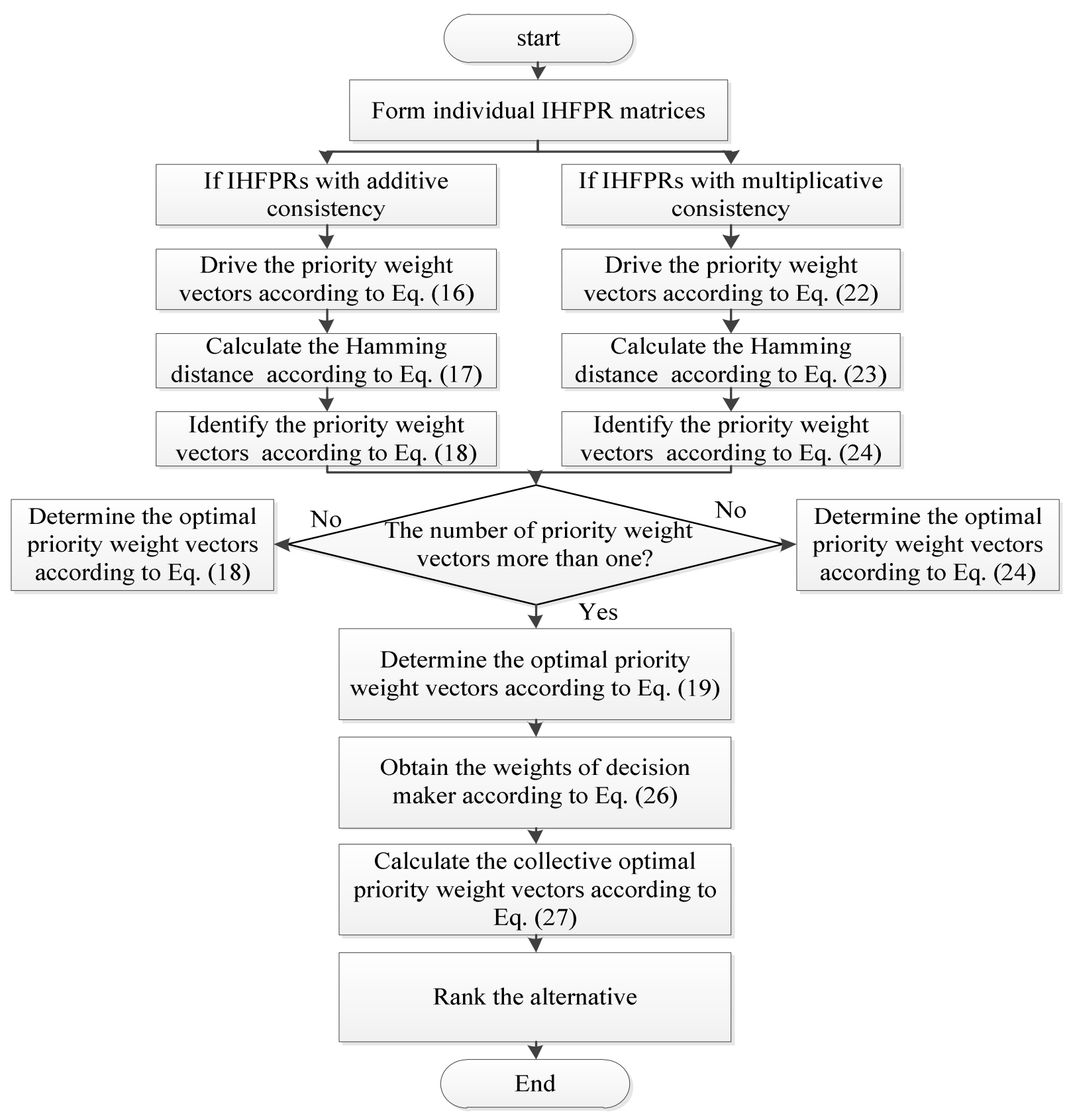

Fig.1. Framework of decision making process with IHFPRs

\section{Illustrative example}

In this section, selection of the most important project to invest problem (adapted from $\mathrm{Xu}$, et al. (2016) and Parreiras, Ekel, Martini, and Palhares (2010)) is provided to illustrate the use of the proposed method, and conjunction with comparative analysis is conducted.

The enterprise's board of directors, which includes three members $e_{k}, k=1,2,3$, named three decision makers, 
have to plan the development of strategy initiatives for the following several years. Suppose that there are three possible projects, denoted as: (1) $a_{1}$ medical intelligent logistics; (2) $a_{2}$ port intelligent logistics; and (3) $a_{3}$ cold chain intelligent logistics, to be evaluated. It is necessary to compare these projects in order to select the most important from the point view of their importance, taking into account four criteria suggested by the balanced scored methodology from the perspectives of: (1) learning and growth; (2) financial; (3) internal business process; and (4) the customer satisfaction. First, three decision makers are asked to give their opinion relative to each project. Because of the uncertainty of the criteria, it is difficult for the decision makers to use just one value to provide their evaluation values. To facilitate the elicitation of their evaluation values, HFPR is just an effective tool to deal with such situations. Furthermore, some decision makers may be have limited expertise and lacking in knowledge related to the problem domain, and thus, these decision makers provide their evaluation with IHFPRs, as demonstrated in matrices 1-3.

Take the evaluation values $\{0.2,0.3\}$ and $\varphi$ from decision maker $e_{1}$ for example. The decision maker $e_{1}$ is hesitant two possible values 0.2 and 0.3 when assesses the alternatives $a_{2}$ to $a_{3}$, and cannot determine which one is the best. In such case, the evaluation value can be modeled by a HFE $\{0.2,0.3\}$. And $\varphi$ means decision maker $e_{1}$ cannot provide any evaluation value owing to lack of background knowledge when assesses the alternatives $a_{1}$ to $a_{3}$. Other entries, that is, HFEs, in matrices $1-3$ are similarly explained.

$$
\begin{gathered}
R_{1}=\left[\begin{array}{ccc}
\{0.5\} & \{0.6\} & \varphi \\
\{0.4\} & \{0.5\} & \{0.2,0.3\} \\
\varphi & \{0.7,0.8\} & \{0.5\}
\end{array}\right] \quad, \quad R_{2}=\left[\begin{array}{ccc}
\{0.5\} & \varphi & \{0.3,0.4\} \\
\varphi & \{0.5\} & \{0.3\} \\
\{0.6,0.7\} & \{0.7\} & \{0.5\}
\end{array}\right] \quad, \quad \text { and } \\
R_{3}=\left[\begin{array}{ccc}
\{0.5\} & \{0.3,0.4\} & \{0.4\} \\
\{0.6,0.7\} & \{0.5\} & \varphi \\
\{0.6\} & \varphi & \{0.5\}
\end{array}\right] .
\end{gathered}
$$

\subsection{Illustration of the proposed method}

The procedures for determining the most important project using the proposed method is discussed below.

Case 1: Suppose that all individual IHFPRs with additive consistency

Step 1: Form individual IHFPR matrices.

All individual IHFPR matrices have been provided, as demonstrated in matrices 1-3. 
Step 2: Derive the priority weight vectors.

Suppose that the decision makers' acceptable deviation value $\varsigma=0.5$. According to Eq. (16), for decision maker $e_{1}$, we have:

$$
\begin{array}{ll}
\max & z^{1}=\varphi \\
\text { s.t. } & \left\{\begin{array}{l}
0.5+\frac{1}{2}\left(w_{1}^{1}-w_{2}^{1}\right)+0.5-0.6 \geq 0.5 \varphi \\
0.5+\frac{1}{2}\left(w_{2}^{1}-w_{3}^{1}\right)+0.5-\left(0.2 \alpha_{23}^{1}+0.3 \alpha_{23}^{2}\right) \geq 0.5 \varphi \\
0.5-\frac{1}{2}\left(w_{1}^{1}-w_{2}^{1}\right)-0.5+0.6 \geq 0.5 \varphi \\
0.5-\frac{1}{2}\left(w_{2}^{1}-w_{3}^{1}\right)-0.5+\left(0.2 \alpha_{23}^{1}+0.3 \alpha_{23}^{2}\right) \geq 0.5 \varphi \\
w_{1}^{1}+w_{2}^{1}+w_{3}^{1}=1 \\
\alpha_{23}^{1}+\alpha_{23}^{2}=1 \\
\alpha_{23}^{s}=0 \vee 1, \quad s=1,2 \\
\alpha_{23}^{1}=1
\end{array}\right.
\end{array}
$$

By solving this optimization model, we obtain:

$z^{1}=0.3 ; w_{1}^{1}=0.9, \quad w_{2}^{1}=0$ and $w_{3}^{1}=0.1 ; \alpha_{23}^{1}=1$.

By replacing $\alpha_{23}^{2}=1$ with $\alpha_{23}^{1}=1$ into above optimization model, we obtain:

$z^{2}=0.4 ; \quad w_{1}^{2}=0.8, \quad w_{2}^{2}=0$ and $w_{3}^{2}=0.2 ; \quad \alpha_{23}^{2}=1$.

Similarly, for decision maker $e_{2}$, we can obtain

$z^{1}=0.1 ; w_{1}^{1}=0.5, w_{2}^{1}=0.5$ and $w_{3}^{1}=0 ; \alpha_{13}^{1}=1$.

$z^{2}=0.2 ; w_{1}^{2}=0.6, w_{2}^{2}=0.4$ and $w_{3}^{2}=0 ; \quad \alpha_{13}^{2}=1$.

Moreover, for decision maker $e_{3}$, we can obtain

$z^{1}=0.2 ; w_{1}^{1}=0, w_{2}^{1}=0.4$ and $w_{3}^{1}=0.6 ; \alpha_{12}^{1}=1$.

$z^{2}=0.3 ; \quad w_{1}^{2}=0, w_{2}^{2}=0.5$ and $w_{3}^{2}=0.5 ; \alpha_{12}^{2}=1$.

Step 3: Derive the optimal priority weight vector. 
First, Utilize Eq. (17) to obtain the distance between $w^{k}$ and $R_{k}$, for decision maker $e_{1}$, we have: $d_{1}\left(w^{1}, R_{1}\right)=\frac{1}{2}\left(\left|\frac{1}{2}(0.9-0)+0.5-0.6\right|+\left|\frac{1}{2}(0-0.1)+0.5-0.2\right|\right)=0.3$ and $d_{1}\left(w^{2}, R_{1}\right)=0.2$. Since there is only one priority weight vector including in Eq. (18), then the optimal priority weight vector can be determined as follows: $w^{1 *}=(0.8,0,0.2)$

Similarly, for decision maker $e_{2}$, we can obtain: $d_{1}\left(w^{1}, R_{2}\right)=0.45$ and $d_{1}\left(w^{2}, R_{2}\right)=0.4$. Since there is only one priority weight vector including in Eq. (18), then the optimal priority weight vector can be determined as follows: $w^{2 *}=(0.6,0.4,0)$.

Moreover, for decision maker $e_{3}$, we can obtain: $d_{1}\left(w^{1}, R_{3}\right)=0.1$ and $d_{1}\left(w^{2}, R_{3}\right)=0.15$. Since there is only one priority weight vector including in Eq. (18), then the optimal priority weight vector can be determined as follows: $w^{3 *}=(0,0.4,0.6)$

Step 4: Determine the weights of decision makers.

According to Eq. (26), the weights of decision makers are determined as follows:

$\lambda_{1}=0.33, \lambda_{2}=0.33$ and $\lambda_{3}=0.33$.

Step 5: Compute the collective optimal priority weight vector.

According to Eq. (27), the collective optimal priority weight vector is determined as follows:

$\vartheta_{1}=0.47, \vartheta_{2}=0.27$ and $\vartheta_{3}=0.27$.

Step 6: Rank the alternatives.

Since $\vartheta_{1}>\vartheta_{2}=\vartheta_{3}$, the ranking order of all alternatives is obtained as $a_{1} \mathrm{f} a_{2} \sim a_{3}$. Thus, the most important project to invest is medical intelligent logistics.

Case 2: Suppose that all individual IHFPRs with multiplicative consistency

Step 1' : Form individual IHFPR matrices.

All individual IHFPR matrices have been provided, as demonstrated in matrices 1-3.

Step 2 : Derive the priority weight vectors. 
Suppose that the decision makers' acceptable deviation value $\varepsilon=0.5$. According to Eq. (22), for decision maker $e_{1}$, we have:

$$
\begin{array}{ll}
\max & z^{1}=\pi \\
\text { s.t. } & \left\{\begin{array}{l}
0.5+\frac{w_{1}^{1}}{w_{1}^{1}+w_{2}^{1}}-0.6 \geq 0.5 \pi \\
0.5-\frac{w_{1}^{1}}{w_{1}^{1}+w_{2}^{1}}+0.6 \geq 0.5 \pi \\
0.5+\frac{w_{2}^{1}}{w_{2}^{1}+w_{3}^{1}}-0.2^{\alpha_{23}^{1}} 0.3^{\alpha_{23}^{2}} \geq 0.5 \pi \\
0.5-\frac{w_{2}^{1}}{w_{2}^{1}+w_{3}^{1}}+0.2^{\alpha_{23}^{1}} 0.3^{\alpha_{23}^{2}} \geq 0.5 \pi \\
w_{1}^{1}+w_{2}^{1}+w_{3}^{1}=1 \\
\alpha_{23}^{1}+\alpha_{23}^{2}=1 \\
\alpha_{23}^{s}=0 \vee 1, \quad s=1,2 \\
\alpha_{23}^{1}=1
\end{array}\right.
\end{array}
$$

By solving this optimization model, we obtain:

$z^{1}=1 ; w_{1}^{1}=0.23, w_{2}^{1}=0.15$ and $w_{3}^{1}=0.62 ; \alpha_{23}^{1}=1$.

By replacing $\alpha_{23}^{2}=1$ with $\alpha_{23}^{1}=1$ into above optimization model, we obtain:

$z^{2}=1 ; w_{1}^{2}=0.31, w_{2}^{2}=0.21$ and $w_{3}^{2}=0.48 ; \alpha_{23}^{2}=1$.

Similarly, for decision maker $e_{2}$, we can obtain

$z^{1}=1 ; w_{1}^{1}=0.23, w_{2}^{1}=0.23$ and $w_{3}^{1}=0.54 ; \alpha_{13}^{1}=1$.

$z^{2}=1 ; w_{1}^{2}=0.32, w_{2}^{2}=0.2$ and $w_{3}^{2}=0.48 ; \alpha_{13}^{2}=1$.

Moreover, for decision maker $e_{3}$, we can obtain

$z^{1}=1 ; w_{1}^{1}=0.21, w_{2}^{1}=0.48$ and $w_{3}^{1}=0.31 ; \alpha_{12}^{1}=1$.

$z^{2}=1 ; w_{1}^{2}=0.25, w_{2}^{2}=0.375$ and $w_{3}^{2}=0.375 ; \quad \alpha_{12}^{2}=1$.

Step 3 : Derive the optimal priority weight vector. 
First, Utilize Eq. (23) to obtain the distance between $w^{k}$ and $R_{k}$, for decision maker $e_{1}$, we have: $d_{2}\left(w^{1}, R_{1}\right)=\frac{1}{2}\left(\left|\frac{0.23}{0.23+0.15}-0.6\right|+\left|\frac{0.15}{0.15+0.62}-0.2\right|\right)=0.0052$ and $d_{2}\left(w^{2}, R_{1}\right)=0.004$. Since there is only one priority weight vector including in Eq. (24), then the optimal priority weight vector can be determined as follows: $w^{1 *}=(0.31,0.21,0.48)$.

Similarly, for decision maker $e_{2}$, we can obtain: $d_{2}\left(w^{1}, R_{2}\right)=0.0013$ and $d_{2}\left(w^{2}, R_{2}\right)=0.0029$. Since there is only one priority weight vector including in Eq. (24), then the optimal priority weight vector can be determined as follows: $w^{2 *}=(0.23,0.23,0.54)$.

Moreover, for decision maker $e_{3}$, we can obtain: $d_{2}\left(w^{1}, R_{3}\right)=0.004$ and $d_{2}\left(w^{2}, R_{3}\right)=0$. Since there is only one priority weight vector including in Eq. (24), then the optimal priority weight vector can be determined as follows: $w^{3 *}=(0.25,0.375,0.375)$.

Step 4 : Determine the weights of decision makers.

According to Eq. (26), the weights of decision makers are determined as follows:

$$
\lambda_{1}=0.33, \lambda_{2}=0.33 \text { and } \lambda_{3}=0.33 \text {. }
$$

Step 5 : Compute the collective optimal priority weight vector.

According to Eq. (27), the collective optimal priority weight vector is determined as follows:

$\vartheta_{1}=0.26, \vartheta_{2}=0.27$ and $\vartheta_{3}=0.47$.

Step 6': Rank the alternatives.

Since $\vartheta_{3}>\vartheta_{2}>\vartheta_{1}$, the ranking order of all alternatives is obtained as $a_{3} \mathrm{f} a_{2} \mathrm{f} a_{1}$. Thus, the most important project to invest is cold chain intelligent logistics.

\subsection{Comparative analysis and discussion}

To validate the feasibility of the proposed method, we conducted a comparative study with other method based on the same illustrative example. 
$\mathrm{Xu}$, et al. (2016) first proposed the concept of IHFPR, and then introduced the concept of additive consistent IHFPR and multiplicative consistent IHFPR. Moreover, to obtain the priority vector of an IHFPR, two goal programming models are developed based on additive consistency and multiplicative consistency respectively. Finally, these two goal programming models have been extended to obtain the collective priority vector of several IHFPRs. To better comparison, the results obtained by Xu, et al. (2016)'s methods and the proposed methods are summarized in Table 2. The detailed calculation process of Xu, et al. (2016)'s methods can be found in Xu, et al. (2016).

Table 2: The ranking results of the different methods

\begin{tabular}{|c|c|c|c|c|c|}
\hline \multirow[t]{2}{*}{ Methods } & & \multicolumn{3}{|c|}{ Ranking values } & \multirow[t]{2}{*}{ Ranking results } \\
\hline & & $\vartheta_{1}$ & $\vartheta_{2}$ & $\vartheta_{3}$ & \\
\hline $\mathrm{Xu}, \quad$ et al. & additive consistency & 0.3184 & 0.2045 & 0.4773 & $a_{3} \mathrm{f} a_{1} \mathrm{f} a_{2}$ \\
\hline (2016)'s methods & multiplicative consistency & 0.3333 & 0.2333 & 0.4333 & $a_{3} \mathrm{f} \quad a_{1} \mathrm{f} \quad a_{2}$ \\
\hline The proposed & additive consistency & 0.47 & 0.27 & 0.27 & $a_{1} \mathrm{f} \quad a_{2}: a_{3}$ \\
\hline methods & multiplicative consistency & 0.26 & 0.27 & 0.47 & $a_{3} \mathrm{f} \quad a_{2} \mathrm{f} \quad a_{1}$ \\
\hline
\end{tabular}

As shown in Table 2, it can be seen that there are some differences in the ranking results. In $\mathrm{Xu}$, et al. (2016)'s methods, the ranking results are the same, but the ranking values are different when considering different consistency. In the proposed methods, both the ranking results and ranking values are different when considering different consistency. It can be easily found that the best alternative obtained from Xu, et al. (2016)'s methods are the same as the proposed method with multiplicative consistency, but the ranking values are different. The possibility reasons for the inconsistency are explained as follows. The consistency definitions are different. In Xu, et al. (2016)'s methods, the consistency definitions based on one FPR derived from IHFPR, that is, optimistic consistency, while the proposed methods the consistency definitions based on each value in HFEs, they considering some possible FPRs derived from IHFPR. The consistency considers all the evaluation information, and neither adds values into HFEs nor removes values from HFEs. It can then avoid information loss and distort. Compare to Xu, et al. (2016)'s method, they only consider some evaluation information, based on this fact, the ranking result obtained from the proposed method seems more reasonable. Moreover, the objective functions are different. In Xu, et al. (2016)'s methods, the objective 
functions constructed based on minimizing the deviation from the target of the goal. However, the proposed methods focus on maximizing the parameter of satisfaction degree. The different perspectives for solving the problems lead to different decision-making results, but the proposed methods take the decision makers' satisfaction degree into account, this is more suitable for solving decision-making problems in some backgrounds (Z. Zhang \& Chen, 2021b).

Furthermore, we provide a possible reason why the different best alternatives are obtained by the proposed methods with different consistency. The additive consistency shows the additive transitivity of the three related judgement, while multiplicative consistency shows the multiplicative transitivity of the three related judgement. Different focus will result in different ranking results. In addition, for the proposed methods with different consistency, lots of weight vectors of criteria are derived. For a better comparison, the results are summarized in Table 3.

Table 3: The weights of criteria derived from the proposed methods with different consistency

\begin{tabular}{llllll}
\hline \multirow{2}{*}{$\begin{array}{l}\text { The proposed methods with different } \\
\text { consistency }\end{array}$} & Decision makers & \multicolumn{2}{l}{ Weight vectors } & \multicolumn{2}{l}{ Objective } \\
\cline { 2 - 5 } function value
\end{tabular}

As shown in Table 3, it can be seen that different weight vectors are obtained for the proposed methods with different consistency. This is consistent with our previous view. In practical decision-making problems, one can determine the method according to the satisfaction value. Furthermore, for different decision makers, since the 
evaluation information is different, weight vectors of criteria derived from them are different, it is necessary consider the weights of decision makers when collecting the priority weight vectors. Unfortunately, the method of determining the weights of decision makers does not consider in Xu, et al. (2016)'s method.

According to the comparison analysis, the method proposed in this study has the following advantages over other existing methods.

(1) Two decision-making models are developed in view of the proposed additive consistency and multiplicative consistency measures. These methods directly utilize the original judgement of evaluation information to make decision without adjusting them. It can then avoid information loss and distort, and the ranking result obtained from the proposed method seems more reasonable.

(2) The proposed methods take into account the decision makers' satisfaction degree. The proposed method is superior to the decision-making methods based on acceptably consistent preference relations because it can omit the procedure to repair the inconsistent preference relations.

(3) The method of determining the weights of decision makers is developed. This method makes full use of the evaluation information of decision-makers.

\section{Conclusion}

This paper develops decision-making models based on decision makers' satisfaction degree with IHFPR. First, the consistency measures from the perspectives of additive and multiplicative consistent IHFPR are defined. Then, two decision-making models are developed in view of the proposed additive and multiplicative consistency measures. Second, a square programming model is developed to obtain the decision makers' weights. Finally, a procedure for MCDM problems with IHFPR is given, and an illustrative example in conjunction with comparative analysis is conducted.

The present study provides several significant contributions for MCDM problems with IHFPR. They are summarized as follows: (1) A new concept of additive and multiplicative consistent IHFPR is proposed, respectively. The main feature of them is that they consider all the evaluation information including in HFEs, and neither add values into HFEs nor remove values from HFEs. They can avoid information loss and distort. (2) Two decision-making models are developed based on the proposed additive and multiplicative consistency measures. The main characteristic of the constructed models is that these methods consider the decision makers' satisfaction degree. (3) A square 
programming model is developed to obtain the decision makers' weights, which is utilized the optimal priority weight vectors information derived from individual IHFPR matrices. In our future research, the proposed methods are extended to hesitant fuzzy linguistic preference relation and applied the proposed methods to solve other practical MCDM problems.

\section{Acknowledgement}

This work was supported by This work was supported by the National Natural Science Foundation of China (Nos.72061026), the Natural Science Foundation of Guangxi (Nos. 2020GXNSFAA297239), the Science and Technology Plan of Guangxi (gui ke AD20238006). The National Natural Science Foundation of China (Nos.61866006), Guangxi innovation-driven development of special funds project (gui ke AA17204091), the Natural Science Foundation of Guangxi (Nos. AB17292095), the Research Funds for the Guangxi University Xingiian College of Science and Liberal Arts (Nos. Y2018ZKT01) and Promotion project of Middle-aged and Young Teachers' Basic Scientific Research Ability in Universities of Guangxi (No. 2019KY0963).

\section{Compliance with Ethical Standards}

Conflict of Interest: Authors Jian Li, Li-li Niu, Qiongxia Chen and Zhong-xing Wang declare that they have no conflict of interest.

Ethical approval: This article does not contain any studies with human participants performed by any of the authors.

\section{References}

Ding, R. X., Palomares, I., Wang, X., Yang, G.-R., Liu, B., Dong, Y., Herrera-Viedma, E., \& Herrera, F. (2020). Large-Scale decision-making: Characterization, taxonomy, challenges and future directions from an Artificial Intelligence and applications perspective. Information Fusion, 59, 84-102.

Dong, Y., Liu, W., Chiclana, F., Kou, G., \& Herrera-Viedma, E. (2019). Are incomplete and self-confident preference relations better in multicriteria decision making? A simulation-based investigation. Information Sciences, 492, 40-57.

Gong, J.-W., Liu, H.-C., You, X.-Y., \& Yin, L. (2021). An integrated multi-criteria decision making approach with linguistic hesitant fuzzy sets for E-learning website evaluation and selection. Applied Soft Computing, 102, 107118 . 
Khalid, A., \& Beg, I. (2017). Incomplete hesitant fuzzy preference relations in group decision making. International Journal of Fuzzy Systems, 19, 637-645.

Li, J., Wang, J. Q., \& Hu, J. H. (2019). Consensus building for hesitant fuzzy preference relations with multiplicative consistency. Computers \& Industrial Engineering, 128, 387-400.

Liu, H., Xu, Z., \& Liao, H. (2016). The Multiplicative Consistency Index of Hesitant Fuzzy Preference Relation. IEEE Transactions on Fuzzy Systems, 24, 82-93.

Liu, J., Li, H., Huang, B., Liu, Y., \& Liu, D. (2021). Convex combination-based consensus analysis for intuitionistic fuzzy three-way group decision. Information Sciences, 574, 542-566.

Liu, X., Wang, Z., Zhang, S., \& Garg, H. (2021). Novel correlation coefficient between hesitant fuzzy sets with application to medical diagnosis. Expert Systems with Applications, 183, 115393.

Meng, F., \& An, Q. (2017). A new approach for group decision making method with hesitant fuzzy preference relations. Knowledge-Based Systems, 127, 1-15.

Meng, F., \& Chen, S.-M. (2021). A framework for group decision making with multiplicative trapezoidal fuzzy preference relations. Information Sciences, DOI: 10.1016/j.ins.2021.1007.1045.

Meng, F., Chen, S.-M., \& Tang, J. (2020). Group decision making based on acceptable multiplicative consistency of hesitant fuzzy preference relations. Information Sciences, 524, 77-96.

Mishra, A. R., Rani, P., Krishankumar, R., Ravichandran, K. S., \& Kar, S. (2021). An extended fuzzy decision-making framework using hesitant fuzzy sets for the drug selection to treat the mild symptoms of Coronavirus Disease 2019 (COVID-19). Applied Soft Computing, 103, 107155.

Orlovsky, S. A. (1978). Decision-making with a fuzzy preference relation. Fuzzy Sets \& Systems, 1, 155-167.

Parreiras, R. O., Ekel, P. Y., Martini, J. S. C., \& Palhares, R. M. (2010). A flexible consensus scheme for multicriteria group decision making under linguistic assessments. Information Sciences, 180, 1075-1089.

Rabiee, M., Aslani, B., \& Rezaei, J. (2021). A decision support system for detecting and handling biased decision-makers in multi criteria group decision-making problems. Expert Systems with Applications, 171, 114597.

Ren, P., Hao, Z., Wang, X., Zeng, X., \& Xu, Z. (2020). Decision-making models based on incomplete hesitant fuzzy linguistic preference relation with application to site selection of hydropower stations. IEEE Transactions on 
Engineering Management, 1, 1-12.

Ren, P., Xu, Z., Wang, X., \& Zeng, X.-J. (2021). Group decision making with hesitant fuzzy linguistic preference relations based on modified extent measurement. Expert Systems with Applications, 171, 114235.

Ren, P., Zhu, B., \& Xu, Z. (2018). Assessment of the impact of hydropower stations on the environment with a hesitant fuzzy linguistic hyperplane-consistency programming method. IEEE Transactions on Fuzzy Systems, 26, 2981-2992.

Rodríguez, R. M., Labella, Á., Dutta, B., \& Martínez, L. (2021). Comprehensive minimum cost models for large scale group decision making with consistent fuzzy preference relations. Knowledge-Based Systems, 215, 106780.

Song, Y., \& Li, G. (2019). Handling group decision-making model with incomplete hesitant fuzzy preference relations and its application in medical decision. Soft Computing, 23, 6657-6666.

Tang, J., An, Q., Meng, F., \& Chen, X. (2017). A natural method for ranking objects from hesitant fuzzy preference relations. International Journal of Information Technology \& Decision Making, 16, 1611-1646.

Tang, J., Chen, S.-M., \& Meng, F. (2019). Heterogeneous group decision making in the setting of incomplete preference relations. Information Sciences, 483, 396-418.

Tanino, T. (1984). Fuzzy preference orerings in group decision-making. Fuzzy Sets \& Systems, 12, 117-131.

Torra, V., \& Narukawa, Y. (2009). On hesitant fuzzy sets and decision. In IEEE International Conference on Fuzzy Systems, 2009. Fuzz-Ieee (pp. 1378-1382).

Wan, S., Yuan, H., \& Dong, J. (2021). Decision making with incomplete interval multiplicative preference relations based on stochastic program and interval category. Information Sciences, 570, 403-427.

Wu, P., Zhu, J., Zhou, L., \& Chen, H. (2019). Local feedback mechanism based on consistency-derived for consensus building in group decision making with hesitant fuzzy linguistic preference relations. Computers \& Industrial Engineering, 137, 106001.

Xia, M., \& Xu, Z. (2013). Managing hesitant information in GDM problems under fuzzy and multiplivative referene relations. International Journal of Uncertainty, Fuzziness, and Knowledge-based Systems, 21, 865-897.

Xia, M. M., \& Xu, Z. S. (2011). Hesitant fuzzy information aggregation in decision making. International Journal of Approximate Reasoning, 52, 395-407.

Xie, W., Xu, Z., Ren, Z., \& Herrera-Viedma, E. (2020). A new multi-criteria decision model based on incomplete dual 
probabilistic linguistic preference relations. Applied Soft Computing, 91, 106237.

Xu, Y., Cabrerizo, F. J., \& Herrera-Viedma, E. (2017). A consensus model for hesitant fuzzy preference relations and its application in water allocation management. Applied Soft Computing, 58, 265-284.

Xu, Y., Chen, L., Rodríguez, R. M., Herrera, F., \& Wang, H. (2016). Deriving the priority weights from incomplete hesitant fuzzy preference relations in group decision making. Knowledge-Based Systems, 99, 71-78.

Yazdani, M., Mohammed, A., Bai, C., \& Labib, A. (2021). A novel hesitant-fuzzy-based group decision approach for outsourcing risk. Expert Systems with Applications, 184, 115517.

Zhang, H., Palomares, I., Dong, Y., \& Wang, W. (2018). Managing non-cooperative behaviors in consensus-based multiple attribute group decision making: An approach based on social network analysis. Knowledge-Based Systems, 162, 29-45.

Zhang, Z. (2016). Deriving the priority weights from incomplete hesitant fuzzy preference relations based on multiplicative consistency. Applied Soft Computing, 46, 37-59.

Zhang, Z., \& Chen, S.-M. (2021a). Group decision making based on multiplicative consistency-and-consensus preference analysis for incomplete q-rung orthopair fuzzy preference relations. Information Sciences, DOI: 10.1016/j.ins.2021.1007.1044.

Zhang, Z., \& Chen, S.-M. (2021b). Group decision making based on multiplicative consistency-and-consensus preference analysis for incomplete q-rung orthopair fuzzy preference relations. Information Sciences, DOI: doi.org/10.1016/j.ins.2021.1007.1044.

Zhang, Z., Kou, X., \& Dong, Q. (2018). Additive consistency analysis and improvement for hesitant fuzzy preference relations. Expert Systems with Applications, 98, 118-128.

Zhang, Z., Kou, X., Yu, W., \& Guo, C. (2018). On priority weights and consistency for incomplete hesitant fuzzy preference relations. Knowledge-Based Systems, 143, 115-126.

Zhang, Z., Wang, C., \& Tian, X. (2015a). A decision support model for group decision making with hesitant fuzzy preference relations. Knowledge-Based Systems, 86, 77-101.

Zhang, Z., Wang, C., \& Tian, X. (2015b). Multi-criteria group decision making with incomplete hesitant fuzzy preference relations. Applied Soft Computing, 36, 1-23.

Zhu, B., \& Xu, Z. (2014a). Analytic hierarchy process-hesitant group decision making. European Journal of 
Operational Research, 239, 794-801.

Zhu, B., \& Xu, Z. (2014b). Consistency measures for hesitant fuzzy linguistic preference relations. IEEE Transactions on Fuzzy Systems, 22, 35-45.

Zhu, B., Xu, Z., \& Xu, J. (2014). Deriving a ranking from hesitant fuzzy preference relations under group decision making. IEEE Transactions on Cybernetics, 44, 1328-1337. 